\title{
Notes on the polypores (Polyporaceae) of Ahvenanmaa
}

\author{
Lalli Laine
}

The archipelago of Ahvenanmaa at the south-western corner of Finland differs in many regards from the rest of the country. The climate is warmer and more humid and, phytogeographically, its vascular plant flora contains abundantly southern species not known on the mainland of Finland.

Only a few papers have been published on the mycoflora of Ahvenanmaa (Swedish: Aland) Frey 1944, STENLId 1947, SchulmanN 1960). Polypores (Polyporaceae) have been observed only occasionally. Most of these observations have been published by STENLID (1947, p. 87, 27 notes of 18 species) also including some rare species. FREY (1944, p. 15-16) has given somewhat fewer observations of Polyporaceae than STENLID. In the collections of mycological herbaria of the Universities of Helsinki and Turku and of the Finnish Forest Research Institute, Helsinki, there are only a few samples (some dozens) of Polyporaceae from Ahvenanmaa.

The author has collected polypores in the Ahvenanmaa area during the last two autums (years 1965 and 1966) in connection with his other investigations. I wish express my deep gratitude to Prof. Dr. V. Kujala who has determined the critical and difficult samples and examined the determinations made by me. I wish also to express my thanks to my fellow excursionists, Dr. V. Hintikka and Mr. M. Kaivos as well as to forest officer Bo Högnäs who kindly provided information on many interesting investigation places in Ahvenanmaa.

The following list of species has been arranged alphabetically. The names of the taxa follow in the main that of BONDARTSEv (1953). Some of the names of resupinate species are after Christiansen (1960). All specimens in the following list, which have been collected by myself (marked L.L. \& M.K. or L.L. \& V.H.), are preserved in the Herbarium of the Finnish Forest Research Institute, Helsinki (abbreviation: HFR). I have also studied the polypores from Ahvenanmaa that are preserved in the Herbarium of the University of Helsinki $(\mathrm{H})$ (collected mainly by Prof. Frey and Dr. Schulmann), as well as those in the Herbarium of the University of Turku (TUR). The collections of STENLID (1947) are located in Sweden and have not been studied by the author. The place names, which have been typed spaced out in the following list of species, refer to the names of the parishes (smallest local government areas in Finland). The following abbreviations of the names of persons have been used: L.L.= Lalli Laine, M.K.= Matti Kaivos, O.v.Sch. $=$ Otto von Schulmann, V.H. = Veikko Hintikka, V.K.=Viljo Kujala. The geographical situation of the Ahvenanmaa area is the following: $60^{\circ} 40^{\prime}-59^{\circ} 30^{\prime} \mathrm{N}$. and $19^{\circ}$ $07^{\prime}-21^{\circ} 20^{\prime} \mathrm{E}$.

Abortiporus borealis (Fr.) Bond. et Sing. (Spongipellis borealis).

E cker $\ddot{o}: 3 \mathrm{~km}$ to the south of Skag, on a stump of Picea abies (8. 10. 1966, L.L. \& V.H.). J o m a 1 a: Gottby, on a stump of Picea abies (6. 10. 1965, L.L. \& M.K.).

Amyloporia calcea (Fr.) Christ. (Det. V.K.)

Finström: Mangelbo, on a wooden fence in Ulmus scabra stand (10. 10. 1966, L.L. \& V.H.). F ö g l ö: Sonnboda E., on a fallen trunk of Pinus silvestris (3. 10.1965, L.L. \& M.K.). G e t a : Soltuna, Getaberg, on Pinus silvestris (10.10.1966, L.L. \& V.H.). J o m a la: $1 \mathrm{~km}$ to the north of the church, on Picea abies (10.10. 1966, L.L. \&V.H.). L u m p a r l a n d: Långnäs, on rotten wood (11. 10. 1966, L.L. \& V.H.); Skag, on Pinus silvestris (7. 10. 1966, L.L. \& V.H.)

A. xantha (Fr.) Bond. et Sing. (Poria xantha)

F ög 1 ö: Sonnboda E., on a fallen trunk of Pinus 
silvestris (3. 10. 1965, L.L. \& M.K.); Jyddö, on Pinus silvestris (9. 10. 1966, L.L. \& V.H.). Ge t a : Soltuna, Getaberg, on Pinus silvestris (10.10.1966, L.L. \& V.H.)

Anisomyces odoratus (Wulf.) Pil. (Trametes odorata)

J o m a la: $2 \mathrm{~km}$ to the northwest of önningeby, on a stump of Picea abies (11. 10. 1966, L.L. \& V.H.)

Antrodia mollis (Somm.) Karst. (Trametes mollis) $\mathrm{H}$ a $\mathrm{m} \mathrm{m}$ a rla $\mathrm{nd}: 1,5 \mathrm{~km}$ to the south-east of the church, on a fallen trunk of Populus tremula (8. 10. 1966, L.L. \& V.H.)

Aporpium semisupinum (Berk. et Curt.) Bond. (Det. V.K.)

J o ma la: Ramsholm, on deciduous wood (6. 10. 1965, L.L. \& M.K.)

A. vulgare (Fr. s. Bres.) Bond. et Sing. (Det. V.K.) F inström: Mangelbo, Ulmus scabra stand, on Corylus avellana (10.10. 1966, L.L. \& V.H.)

Bjenkandera adusta (Willd.) Karst. (Polyporus adustus)

$\mathrm{H}$ a m m a r land : $1,5 \mathrm{~km}$ south-east of the church, on Picea abies (8.10.1966, L.L. \& V.H.). J o ma la: Möckelö, on deciduous wood (6.10. 1965, L.L. \& M.K.); Ramsholm, on Alnus glutinosa (8. 10. 1966, L.L. \& V.H.). L e m la n d: Nåtö (15. 9. 1946) and Idholm (22. 9. 1946, G. Stenlid, STENLID 1947). M a aria n hamina: Ytternäs, on a stump of Alnus glutinosa (1. 10. 1965, L.L. \& M.K.); on Fraxinus excelsior (6. 10. 1965, L.L. \&M.K.); Ytternäs (1.9.1955, O.v.Sch. HFR)

Ceraporia incarnata (Fr. s. Bres.) Bond. (Det. V.K.) Eckerö: Skag, on Fraximus excelsior (7. 10. 1966, L.L. \& V.H.). J om a la: Ramsholm, on a fallen Fraxinus excelsior, (8.10.1966, L.L. \& V.H.). L u m p a r la nd: Skag, on Fraxinus excelsior (7. 10. 1966, L.L. \& V.H.)

C. viridans (Berk. et Br.) Donk f. inconstans (Karst.) B. et G. (Det. V.K.)

J o ma la: Möckelö (21.8.1958, O.v.Sch., HFR)

Cerrena unicolor (Bull.) Murr. (Daedalea unicolor)

E ckerö: Skag (12.9.1963, Esteri Kankainen, TUR). Fin ström : Mangelbo, on Betula (7.10. 1965, L.L. \& M.K.); Torrbolstad, on Betula (2. 10. 1965, L.L. \& M.K.). Föglö: Bråttö, on Betula (3. 10. 1965, L.L. \& M.K.). G e t a, Häckböle, on Corylus avellana (25.9. 1942, R. Frey, H). J o m a l a : $2 \mathrm{~km}$ north-west of Önningeby, on Betula (11.10. 1966, L.L. \& V.H.); at the border of Hammarland (21. 9. 1964, Heli Heikkilä, TUR). M a a ri a n h a mina: Ytternäs, on Betula (10. 10 1966, L.L. \& V.H.) Saltvik: Kvarnbo, on a stump of Populus tremula (9.9.1945, Carl Cedercreutz, H)

Chaetoporellus asiaticus (Pil.) Christ. (Det. V.K.) F inström: Mangelbo, Ulmus scabra stand, on rotten wood $(10.10 .1966$, L.L. \& V.H.; 7.10. 1965, L.L. \& M.K.); Bartsgarda, at the border of Saltvik, on Picea abies (5. 10.1965, L.L. \& M.K.)
Ch. Litshaueri (Pil.) Bond. (Det. V.K.)

J o m a la : Möckelö, on rotten wood (6.10.1965, L.L. \& M.K.)

Chaetoporus corticola (Fr.) Bond. et Sing.

Föglö: Jyddö, on a fallen trunk of Quercus robur 9. 10. 1966, L.L. \& V.H. Det. V.K.)

Ch. euporus (Karst.) Bond. et Sing. (Det. V.K.)

Le m lan d: Flaka, on Betula (11.10.1966, L.L. \& V.H.)

Ch. radulus (Pers.) Bond. et Sing. (Det. V.K.)

J o ma la : Möckelö (6. 10. 1965, L.L. \& M.K.)

Coltricia cinnamomea (Jacq.) Murr. (Polyporus cinnamomeus)

E ckerö: on rotten wood (4. 10.1954, O.v.Sch., HFR)

C. perennis (L.) Murr. (Polystictus perennis)

G e t a : Snäckö (28.9.1949, C. Cedercreutz, H)

Coriolellus heteromorphus (Fr.) Bond. et Sing. (Trametes heteromorpha)

Föglö: Bråttö, on a stump of Picea abies (3. 10. 1965, L.L. \& M.K.). G e ta : Bolstaholm, on a stump of Picea abies (30.9. 1942, R, Frey, (det V.K., H). Jomala: in Abiete» (1.9.1881, P. A. Karsten, H); Jomala (2.9.1881, P. A. Karsten, H)

C. serialis (Fr.) Murr. (Trametes serialis)

E ckerö: Storby, on a stump of Picea abies (13. 7. 1935, L. E. Kari, TUR); (25.5. 1955, O.v.Sch., HFR); Skag, on Picea abies (4.10.1965, L.L. \& M.K.). F in s tröm: near the border of Geta, on Picea abies (10.10.1966, L.L. \&V.H.); Mangelbo, Ulmus scabra stand (10. 10. 1966, L.L. \&V.H.); Torrbolstad (2. 10. 1965, L.L. \& M.K.). Föglö: $1 \mathrm{~km}$ to the east of Sonnboda, on Pinus silvestris 9.10 . 1966, L.L. \& V.H.). J o ma la: Möckelö (13.8. 1958, O.v.Sch., HFR); 6. 10. 1965, L.L. \& M.K.); Ramsholm, on Picea abies (4. 10. 1965, L.L. \& M.K.); Ramsholm, on Picea abies 8. 10.1966, L.L. \& V.H.). L e m la nd: Flaka, on Picea abies (8. 10. 1966, L.L. \& V.H.); Nåtö, on stumps of Picea abies (15. 9. 1946, G. Stenlid, Stentid 1947). L u m p a r l a n d: Skag, on Picea abies and Pinus silvestris (7. 10. 1966, L.L. \& V.H.). Maarianhamina: on Picea abies (5. 10.1958, O.v.Sch., HFR)

Coriolus hirsutus (Wulf.) Quél.

Fin s tröm: near the border of Geta, on Populus tremula (10. 10. 1966, L.L. \& V.H.). J o m a la: Ramsholm, on Sorbus aucuparia (5. 10. 1965, L.L. \& M.K.). Lem land: Granholm, on Populus tremula (21.6. 1949, Ernst Häyrén, H)

C. Hoehnelii (Bres.) Bourd. et Galz. (Det. V.K.) Finström: Mangelbo, on rotten wood of Fraxinus excelsior (7.10.1965, L.L. \& M.K.). Joma la : Möckelö (6.10.1965, L.L. \& M.K.); Ramsholm, on Corylus avellana (4. 10. 1965, L.L. \& M.K.)

C. pubescens (Schum.) Quél.

Eckerö: on Betula (7.10.1942, Carl Cedercreutz, Frey 1944; Skag, on Betula (8. 10. 1966, L.L. \& V.H.); Skag, on a standing trunk of Alnus glu- 
tinosa (4. 10. 1965, L.L. \& M.K.). F öglö: Stentorpa, Granboda, on Betula (3.10.1965, L.L. \& M.K.); Jyddö, on Quercus robur (9. 10.1966, L.L. \&V.H.). G e ta : Häckböle, on Alnus (26.9.1942, R. Frey, Frey 1944 as C. velutinus Fr.). J o m a la : kyrkobyn, on Populus tremula, (on old wattled fence) (10.10.1966, L.L. \& V.H.); Ramsholm, on Fraxinus excelsior (8.10. 1966, L.L. \& V.H.). L e m 1 a n d : Knutsboda (19. 7. 1950, O.v.Sch., H); Flaka, on Betula (11.9.1963, Heli Heikkilä, TUR)

Coriolus vaporarius (Fr.) Bond. et Sing. (Poria vaporaria)

Finström: Torrbolstad, on rotten wood (2. 10. 1965, L.L. \& M.K.). Föglö: $1 \mathrm{~km}$ to the east of Sonnboda, on Pinus silvestris (9.10.1966, L.L. \& V.H.). Get a : Soltuna, Getaberg, on Pinus silvestris (10. 10. 1966, L.L. \& V.H.). J o ma la : Möckelö (13. 8. 1958, O.v.Sch., HFR); Möckelö, on Picea abies (6. 10. 1965, L.L. \& M.K.); to the north of the church, on Picea abies (10.10.1966, L.L. \& V.H.); $2 \mathrm{~km}$ to the north-west of önningeby, on Picea abies (11. 10. 1966, L.L. \& V.H.). L u m parla nd: Skag, on Pinus silvestris (7. 10.1966, L.L. \& V.H.)

C. zonatus (Nees) Quél.

E ck e rö: Skag, on Populus tremula (8. 10. 1966, L.L. \& V.H.). F inst $\mathrm{röm}$ : Torrbolstad, on Betula (2. 10. 1965, L.L. \& M.K.). G e ta: Bolstaholm (30. 9. 1942, R. Frey, Frey 1944). J o m a l a : Möckelö (6. 10. 1965, L.L. \& M.K.); South of Möckelö, Grägersö, on Populus tremula (6.10.1965, L.L. \& M.K.). L e m la nd: Nåtö, on Picea abies (8.6. 1937, L.E. Kari, TUR). M a a ri a $\mathrm{h}$ a m in a : Ytternäs, on Alnus glutinosa (9.6.1937, L. E. Kari, TUR); on Picea abies (20.9. 1958, O.v.Sch. HFR); (6. 10. 1965, L.L. \& M.K.)

Daedalea quercina (L.) Fr.

Eckerö: on Quercus (30.5.1955, O.v.Sch., HFR); Skag, on Quercus robur (8. 10.1966, L.L. \& V.H.). Föglö: Jyddö, on Quercus robur (9.10. 1966, L.L. \& V.H.); Jyddö, on a stump of Quercus robur (9.10.1966, L.L. \& V.H.). G e ta: Östergeta, on Quercus stumps, (25.9. 1942, R. Frey, FreY 1944)

Daedaleopsis confragosa (Bolt. ex Fr.) Schroet. f. rubescens (Alb. et Schw.) Donk (Trametes rubescens) Very rare in Finland. L e m land: Apalholmen (19.9. 1946, G. Stenlid, det S. Lundell, STENLID 1947)

Fibuloporia mollusca (Pers.) Bond. et Sing.

E cke r ö: $3 \mathrm{~km}$ to the south of Skag, on rotten wood of Picea abies (8. 10. 1966, L.L. \& V.H.). F i n ström: near the borden of Geta, on Picea abies (10. 10.1966, L.L. \& V.H.). F öglö: Bråttö, on Pinus silvestris (3. 10. 1965, L.L. \& V.H.). J o m a l a : $2 \mathrm{~km}$ to the north-west of Onningeby, on fallen trunks of Betula and Picea abies (11. 10.1966, L.L. \& V.H.)

Fistulina hepatica (Schaeff.) Fr.

E ckerö: Skag, on Quercus robur (8. 10.1966, L.L. \& V.H.). Föglö: Jyddö, on Quercus robur (9. 10. 1966, L.L. \& V.H.). Ge ta: on Quercus robur (25. 9. 1942, R. Frey, H)
Fomes fomentarius (L.) Gill.

E cke rö: Skag, on Betula (4.10.1965, L.L. \& M.K.). Geta: on Betula (29.9. 1942, R. Frey, H). Jomala: Gottby, on Betula (5.10.1965, L.L. \& M.K.); Kyrkobyn, on Betula (10.10. 1966, L.L. \& V.H.). Le m la nd: Flaka, on Betula (11.10.1966, L.L. \& V.H.). Lumpa r land: Skag, on Betula (7. 10. 1966, L.L. \& V.H.). M a a ria $n$ h a m in a: Ytternäs, on Betula (1.9.1958, O.v.Sch., HFR)

Fomitopsis annosa (Fr.) Karst. (Fomes annosus) E cke rö: Skag, on a stump of Picea abies (4. 10. 1965, L.L. \& M.K.). F in st röm : Torrbolstad, frequent on the stumps of Picea abies and on the roots of one living tree (2. 10. 1965, L.L. \& M.K.). $\mathrm{Ham} \mathrm{marland:} 1,5 \mathrm{~km}$ to the southeast of the church, on Picea abies (8.10.1966, L.L. \& V.H.). J o ma l a : Möckelö, (23.7. 1958, O.v.Sch., HFR); Möckelö, on Picea abies (6. 10.1965, L.L. \& M.K.); Ramsholm (14.9.1958, O.v.Sch., HFR); Ramsholm, on the stumps of Picea abies (5.10.1965, L.L. \& M.K.); Gottby, on the stumps of Picea abies (5. 10 . 1965 , L.L. \& M.K.); $1 \mathrm{~km}$ to the north of the church, on Picea abies (10.10.1966, L.L. \& V.H.); $2 \mathrm{~km}$ to the north-west of Önningeby, on a stump of Picea abies (11. 10. 1966, L.L. \& V.H.). L u m p a rl a n d : Skag, on Picea abies (7.10.1966, L.L. \& V.H.). Maarianhamina: Ytternäs, on a stump of Picea abies, at the bases of small trees of Pinus silvestris and of standing Juniperus communis (1. 10 . 1965, L.L. \& M.K.). S u n d: Kastelholm, on a stump of Picea abies (5. 10. 1965, L.L. \& M.K.)

\section{F. pinicola (Sw.) Karst. (Polyporus pinicola)}

Finström: Torrbolstad, on Picea abies (2. 10. 1965, L.L. \& M.K.). Get a : on Picea abies (29. 9. 1942, R. Frey, H). J o m a l a : Möckelö, on a dead trunk of Picea abies (25.9.1958, O.v.Sch., HFR); Kyrkobyn, on Picea abies (10. 10. 1966, L.L. \& V.H.); $2 \mathrm{~km}$ to the north-west of Önningeby, on Picea abies (11. 10. 1966, L.L. \& V.H.). L e m l a nd: Lemböte (23. 9. 1946, G. Stenlid, Stenld 1947). L u m p a r l a nd: Skag, on Picea abies (7.10.1966, L.L. \& V.H.). M a a rianhamina: (Mariehamn) (18. 9. 1946, G. Stenlid, Stenlid 1947).

Ganoderma applanatum (Pers.) Pat.

F ögl ö: Bråttö, on Betula (3. 10.1965, L.L. \& M.K.). J o m a la : (2.9. 1881, Herb. P. A. Karsten, $\mathrm{H}$ ); near the church, on a stump of Fraxinus excelsior (7. 10. 1965, L.L. \& M.K.)

Gloeophyllum sepiarium (Wulf.) Karst. (Lenzites sepiaria)

E ck e rö: $3 \mathrm{~km}$ to the south of Skag, on Picea abies (8. 10. 1966, L.L. \& V.H.). F in s t rö m: Torrbolstad (2. 10.1965, L.L. \& M.K.). Föglö: east of Sonnboda, on Pinus silvestris (3. 10. 1965, L.L. \& M.K.); $1 \mathrm{~km}$ to the east of Sonnboda, on Pinus silvestris (9. 10. 1966, L.L. \& V.H.). J o m a 1 a : Gottby, on Picea abies (6. 10.1965, L.L. \& M.K.); Ramsholm, on a stump of Picea abies (5. 10. 1965, L.L. \& M.K.); Ramsholm, on an old burned pole of Pinus silvestris (8. 10. 1966, L.L. \& V.H.); Kyrkobyn, on Picea abies (10.10. 1966, L.L. \& V.H.); $2 \mathrm{~km}$ to the northwest of önningeby, on a fallen trunk of Picea abies (11. 10. 1966, L.L. \& V.H.). L e m l a n d: Inre Körsö (17. 9. 1946, G. Stenlid, Stenlm 1947). L u m par - 
I a nd: Skag, on Picea abies (7.10.1966, L.L. \& V.H.)

Gloeoporus amorphus (Fr.) Clem. et Shear (Polyporus amorphus)

Eckerö: Torp, on Pinus silvestris (14.6.1937, L. E. Kari, TUR). Fin ström: Torrbolstad (2. 10. 1965, L.L. \& M.K.). Föglö: east of Sonnboda, on Pinus silvestris (3.10.1965, L.L. \& M.K.); $1 \mathrm{~km}$ to the east of Sonnboda, on Pinus silvestris (9. 10. 1966, L.L. \& V.H.). H a m m a rla n d : $1,5 \mathrm{~km}$ to the southeast of the church, on Picea abies (8. 10. 1966, L.L. \& V.H.). Jo mal a: Ramsholm (6. 10. 1965, L.L. \& M.K.); Möckelö, on Pinus (6. 10.1965, L.L. \& M.K.). L u m pa r land: Skag, on a stump of Picea abies (7. 10. 1966, L.L. \& V.H.)

Hapalopilus nidulans (Fr.) Karst. (Polyporus nidulans)

J o ma la : Ramsholm (4. 10. 1965, L.L. \& M.K. Det. V.K.). L e m la nd: Eskskär, on Sorbus (6.6. 1946, G. Stenlid, det. S. Lundell, StenLid 1947).

H. ochraceo-lateritius (Bond.) Bond. et Sing.

New for Finland. F ö glö: east of Sonnboda, on rotten wood of Pinus silvestris (3. 10.1965, L.L. \& M.K. Det. V.K.).

Hirschioporus abietinus (Dicks.) Donk (Coriolus abietinus)

E cke rö: Storby, Storäng, on Picea abies (11. 6. 1937, L. E. Kari, TUR); Skag, on Picea abies (4. 10. 1965, L.L. \& M.K.); $3 \mathrm{~km}$ to the south of Sonnboda, on Picea abies and Pinus silvestris (9. 10. 1966, L.L. \& V.H.). F in ström: Torrbolstad, on Pinus silvestris and Picea abies (2. 10. 1965, L.L. \& M.K.); near the border of Geta, on Picea abies and Pinus silvestris (10. 10. 1966, L.L. \& V.H.). F ö g l ö : Bråttö, on Pinus silvestris (3. 10. 1965, L.L. \& M.K.); $1 \mathrm{~km}$ to the east of Sonnboda, on Picea abies and Pinus silvestris (9. 10. 1966, L.L. \& V.H.). Ge t a : on Picea abies (28.9.1942, R. Frey, H); Soltuna, Getaberg, on Pinus silvestris (10.10.1966, L.L. \& V.H.). J o$\mathrm{m}$ a la: Gottby, on Picea abies (6.10.1965, L.L. \& M.K.); Ramsholm, on Picea abies (4. 10. 1965, L.L. \& M.K.); $2 \mathrm{~km}$ to the north-west of önningeby, on Pinus silvestris (11.10.1966, L.L. \& V.H.). Le m la n d: Flaka, on Picea abies (11. 10. 1966, L.L. \& V.H.). Lum p a rland: Skag, on Pinus silvestris (7. 10. 1966, L.L. \& V.H.); Långnäs, on Picea abies (11. 10. 1966, L.L. \& V.H.). M a a ri a $\mathrm{n}$ h a m in a: Ytternäs, on Picea abies (1. 10. 1965, L.L. \& M.K.). S und: Kastelholm, on Abies sibirica (5. 10. 1965, L.L. \& M.K.)

H. fusco-violaceus (Ehrenb. ex Fr.) Donk (Irpex fusco-violaccus)

Fögl ̈̈: $1 \mathrm{~km}$ to th east of Sonnboda on Picea abies (9. 10. 1966, L.L. \& V.H.). Jomala: Möckelö, on Pinus silvestris (6. 10. 1965 L.L. \& M.K.); $1 \mathrm{~km}$ to the north of the church, on Pinus silvestris (10. 10. 1966, L.L. \& M.K.)

Inonotus obliquus (Pers.) Pil. (Poria obliqua)

E ckerö: Skag, on Betula (4. 10. 1965, L.L. \& M.K.). F ögl ö: Bråttö, on Betula (3. 10. 1965, L.L. \& M.K.). Jom a la: Gottby, on Alnus glutinosa (6. 10. 1965, L.L. \& M.K.); $1 \mathrm{~km}$ to the north of the church, on Betula (10.10.1966, L.L. \& V.H.);
$2 \mathrm{~km}$ to the north-west of Önningeby, on Betula (11. 10. 1966, L.L. \& V.H.). L u m p a r l a n d: Skag, on Betula (7. 10.1966, L.L. \& V.H.). M a a ria nh a m in a : Ytternäs, on Betula (10.10.1966, L.L. \& V.H.)

\section{I. radiatus (Sow.) Karst. (Polyporus radiatus)}

F inström: Torrbolstad, on Alnus glutinosa and Betula pubescens (2.10.1965, L.L. \& M.K.). Föglö: near Granboda, on Alnus glutinosa (3. 10. 1965, L.L. \& M.K.); Jyddö, on Alnus glutinosa (?) (9. 10.1966, L.L. \& V.H.). J o mala: Gottby, on Alnus glutinosa (5. 10. 1965, L.L. \& M.K.); Ramsholm, on Alnus glutinosa (6. 10. 1965, L.L. \& M.K.); Ramsholm, on Alnus glutinosa and Corylus avellana (8. 10. 1966, L.L. \& V.H.). L e m I a n d: Flaka, on Alnus glutinosa (11.10.1966, L.L. \& V.H.); Flakan (11. 9. 1963, Esteri Kankainen, TUR). Jungfruskär (17. 6. 1945), Nåtö (15. 9. 1946, and Yttre Korsö (24. 9. 1946, G. Stenlid, Stenlid 1947). L u m p a r la nd: Skag, on Alnus glutinosa (7.10.1966, L.L. \& V.H.). Maarianhamina: by the cemetery, on a stump of Alnus glutinosa (5.10. 1965, L.L. \& M.K.); Ytternäs, on Alnus glutinosa and Corylus avellana (10. 10. 1966, L.L. \& V.H.)

\section{Ischnoderma resinosum (Fr.) Karst.}

Finström: Torrbolstad, on a fallen trunk of Pinus silvestris (2. 10.1965, L.L. \& M.K.). F öglö: Bråttö, on a fallen trunk of Picea abies (3. 10.1965, L.L. \& M.K.). G e ta: Soltuna, Getaberg, on Picea abies and Pinus silvestris (10. 10. 1966, L.L. \& V.H.). Jomala: $1 \mathrm{~km}$ to the north of the church, on Picea abies (10.10. 1966, L.L. \& V.H.). M a a ri a n h a mina: ( .10 .1958 , O.v.Sch., HFR); Ytternäs, (20.9. 1964, Paavo Kallio, TUR); Ytternäs, on Picea abies (10. 10. 1966, L.L. \& V.H.)

Laetiporus sulphureus (Bull.) Bond. et Sing. (Polyporus sulphureus)

J o ma la: near the church, on an old standing Populus alba tree (7. 10. 1965, L.L. \& M.K.). Ge ta : Östergeta, on Quercus robur (25.9.1942, R. Frey, FREY 1944)

Lenzites betulina (L.) Fr.

L e m l a n d: Nåtö (15.9. 1946) and Apalholmen (19.9. 1946, G. Stenlid, Stenlid 1946)

Merulioporia taxicola (Pers.) Bond. et Sing.

G e t a : Soltuna, Getaberg, on Pinus silvestris (10. 10. 1966, L.L. \& V.H.)

Merulius tremellosus (Schrad.) Fr.

Föglö: Jyddö, on Betula (9.10.1966, L.L. \& V.H.). M a a ria $\mathrm{n}$ h a m in a: Ytternäs, on Betula (10. 10. 1966, L.L. \& V.H.). L e m l a n d: Nåtö, on fallen Betula (14.9.1946, G. Stenlid, Stenlm 1947)

Oxyporus obducens (Pers. ex Fr.) Donk (Det. V.K.) Ma a rianhamina: Espholm, on Sorbus aucuparia (4. 8. 1938, E. Häyrén, H)

O. populinus (Schum. ex Fr.) Donk (Polyporus populinus)

Eckerö: Skag (12.9.1963, Esteri Kankainen, TUR); Skag, on Alnus glutinosa, Fraxinus excelsior (abundant) and Sorbus aucuparia (4. 10. 1965, L.L. \& M.K.); Skag, on Fraxinus excelsior (8.10.1966, 
L.L. \& V.H.). Fin ström: Mangelbo, on Fraxinus excelsior (7. 10. 1965, L.L. \& M.K.). J o ma la : Ramsholm, on Fraxinus excelsior (6.6.1937, L. E. Kari, TUR). L e m la n d : Nåtö, on living Acer (18. 9. 1946, G. Stenlid, Stenlid 1947). M a a ri a nh a m in a: Ytternäs, on Fraxinus excelsior (6.10. 1965, L.L. \& M.K.); Ytternäs, on Betula, Fraxinus excelsior and Sorbus aucuparia (10.10.1966, L.L. \& V.H.). Sund: by the church, on Acer platanoides (5. 10. 1965, L.L. \& M.K.)

Phaeolus Schweinitzii (Fr.) Pat.

Eckerö: Björnhuvud (21.9. 1964, Paavo Kallio, TUR)

Phellinus conchatus (Pers.) Quél.

Eckerö: Skag, on Prunus padus (4.10.1965, L.L. \& M.K.). Fögl ö: near Stentorpa, on Salix caprea (3. 10. 1965, L.L. \& M.K.). J o ma la : Möckelö (6.6.1937, L. E. Kari, TUR). Lu m par la nd: Skag, on Salix caprea (7.10.1966, L.L. \& V.H.). M a a i a n ha min a: Ytternäs (21.9.1964, Paavo Kallio, TUR)

Ph. ferruginosus (Schrad.) B. et G. Very rare in Finland.

Finström: Mangelbo, on Corylus avellana (7. 10. 1965, L.L. \& M.K.); Mangelbo, Ulmus scabra grove, on Corylus avellana and Ulmus scabra (10. 10. 1966, L.L. \& V.H.). M a a ria $\mathrm{nh}$ a mina: Ytternäs, on Crataegus (6.10.1965, L.L. \& M.K.) rius)

Ph. igniarius (L.) Quél. (Polyporus, Fomes ignia-

Eckerö: Skag (4. 10. 1965, L.L. \& M.K.). Fög 1ӧ: near Granboda, on Salix caprea (3.10.1965, L.L. \& M.K.). Geta, Bolstaholm, on Alnus glutinosa (30.9. 1942, R. Frey, H). J o m a l a : Gottby, on Betula (5.10.1965, L.L. \& M.K.); by the church, on Populus (5.10.1965, L.L. \& M.K.). Köka r: Idö, on living Salix (caprea?) (23. 8. 1954, Ulla Cedercreutz). L e m la nd: Lemströms kanal, on Sorbus hybrida (3. 10. 1965, L.L. \& M.K.); Flaka, on Alnus glutinosa (11.10.1966, L.L. \& V.H.). M a a ri a n$\mathrm{h}$ a m ina: Ytternäs, on Betula (10.10.1966, L.L. \& V.H.). Saltvik: Kvarnbo, on living Fraxinus excelsior (23.8.1954, Ulla Cedercreutz); by the church, on Sorbus suecica (5. 10. 1965, L.L. \& M.K.)

Ph. isabellinus (Fr.) B. et G.

F inström: Bartsgårda, near the border of Saltvik, on rotten wood (5.10.1965, L.L. \& M.K.). $\mathrm{H}$ a $\mathrm{mm}$ a rla $\mathrm{nd}: 1,5 \mathrm{~km}$ to the southeast of the church, on Picea abies (8.10.1966, L.L. \& V.H.). $\mathrm{L} \mathrm{u} \mathrm{m}$ p a r 1 a n d: Skag, on an old wattled fence ( $P i$ cea abies) (7. 10. 1966, L.L. \&V.H.)

Ph. laevigatus (Fr.) B. et G.

Jo mala: $2 \mathrm{~km}$ to the north-west of Önningeby, on Betula (11. 10.1966, L.L. \& V.H.). L u m parland: Skag, on an old fallen trunk of $B e$ tula (7. 10. 1966, L.L. \& V.H.)

Ph. pini (Thore ex Fr.) Pil. (Trametes pini)

Eckerö: on Pinus silvestris (14.3. 1960, (Anonymous, HFR). Föglö: near the church, on the trunk of a living Pinus silvestris (3.10.1965, L.L. \& M.K.). J o mala: $2 \mathrm{~km}$ to the north-west of önningeby, on Pinus silvestris (11.10.1966, L.L. \&
V.H.). L e m land: Yttre Korsö, on Pinus silvestris (24.9.1946, G. Stenlid, Stenlid 1947) Pil.

Ph. pini (Thore ex Fr.) Pil. var. abietis (Karst.)

Finström: Torrbolstad, on Picea abies (2. 10. 1965, L.L. \& M.K.). Jom a la: Gottby, on Picea abies (6. 10.1965, L.L. \& M.K.); $2 \mathrm{~km}$ to the northwest of Önningeby, on Picea abies (11. 10. 1966, L.L. \& V.H.). Le m la nd: Flaka, on rotten wood of Picea abies (11. 10. 1966, L.L. \& V.H.)

Ph. pomaceus (Pers.) Maire (Polyporus igniarius ssp. pomaceus)

J o m a la: near the church, on Prunus sp. (cherry tree) (10.6.1937, O. K. Silkkilä, TUR)

\section{Ph. punctatus (Fr.) Pil.}

J o ma la: Möckelö (11.8. 1958, O.v.Sch., HFR) $\mathrm{Ma}$ a ria $\mathrm{nh}$ a mina: Ytternäs, on Corylus avellana (10. 10. 1966, L.L. \& V.H.)

Ph. robustus (Karst.) B. et G. f. hippophaës Donk Eckerö: Flötan, on Hippophaë rhamnoides (20.6. 1946, G. Stenlid, Stenlm 1947); Skag, on Hippophä̈ rhamnoides (4.10.1965, L.L. \& M.K. and 8. 10. 1966, L.L. \& V.H.). J o ma I a : Espholm, on living Hippophä̈ (10.7. 1945, Ulla Bärlund, HFR); Espholm, Ormgrund, on living Hippophaë rhamnoides (6.7.1945, Ulla Bärlund, HFR); Espholm, Grynnorna, on old Hippophaë rhamnoides branches (27-29. 6. 1947, Ulla Bärlund, HFR); Möckelö (8. 8. 1958, O.v.Sch., HFR); Ramsholm (4. 10. 1965, L.L. \& M.K.). L e m la nd: $2 \mathrm{~km}$ to the south of Flaka, on Hippophaë rhamnoides (11.10.1966, L.L. \& V.H.); Segelgrund, on Hippophä̈ rhamnoides (20. 9. 1946, G. Stenlid, STENLID 1947). M a a ri a n hamina: Ytternäs, on Hippophä̈ rhamnoides (1. 7. 1947 and 22.6.1950, Ulla Bärlund, HFR); Ytternäs, on Hippophaë (15.9. and 20. 9. 1958, O.v.Sch., HFR); Ytternäs, on Hippophä̈ (1.10. and 6.10. 19.65, L.L. \& M.K.)

Ph. tremulae (Bond.) Bond. et Boriss.

Eckerö: Skag, on Populus tremula (4. 10. 1965. L.L. \& M.K.). F inström: Färjsundet, on Populus tremula (28.2. 1965, Bo Högnäs, HFR). J o mala: near the church, on Populus tremula (2. 10. 1965, L.L. \& M.K.). L e m 1 a nd: Granholm, on living Populus tremula (25.6. 1951, Ulla Bärlund, HFR). Saltvik: by the church, on Populus tremula (5. 10. 1965, L.L. \& M.K.)

Phlebiella candidissima (Schw.) Bond. et Sing. F in ström: Torrbolstad, on Picea abies (2. 10. 1965, L.L. \& M.K. Det. V.K.)

Ph. trachyspora (B. et G.) Bond. et Sing. (Lindtneria trachyspora) (Det. V.K.)

Very rare, new for Finland.

Eckerö: Skag, on fallen trunks of Fraxinus excelsior and Alnus glutinosa (4.10. 1965, L.L. \& M.K. and 8.10.1966, L.L. \& V.H.)

Piptoporus betulinus (Bull.) Karst.

E ckerö: Skag, on Betula (4.10.1965, L.L. \& M.K.); Skag, on Betula (8.10.1966, L.L. \& V.H.). F in ström: Färjsundet, on Betula (28.2.1965, Bo Högnäs, HFR). Föglö: Bråttö, on Betula (3. 
10. 1965, L.L. \& M.K.). Geta: on Betula (20.9. 1942, R. Frey, H). J o m a l a : Gottby, on Betula (2. 10. 1965, L.L. \& M.K.); Möckelö, on Betula (6.10 1965 , L.L. \& M.K.); $1 \mathrm{~km}$ to the north of the church, on Betula (10. 10.1966, L.L. \& V.H.). L u m par I a n d: Ramsholm (20.9. 1964, Paavo Kallio, TUR); Skag, on Betula (7. 10. 1966, L.L. \& V.H.)

Podoporia sanguinolenta (Alb. et Schw.) Hoehn. (Det. V.K. 1967)

F in s t r ö m : near the border of Geta, on Pinus silvestris (10.10. 1966, L.L. \& V.H.). Le m lan d: Flaka, on Picea abies (11.10.1966, L.L. \& V.H.)

\section{Polyporus arcularius (Batsch) Fr.}

E cker ö: Skag, on Sorbus aucuparia (4.10. 1965, L.L. \& M.K.); Skag, on Betula (8.10.1966, L.L. \& V.H.). J o m a l a : Ramsholm (6.6. 1937, L. E. Kari, TUR) - f. grisea (Pil.) Lundell. S u n d: Kungsholm (20.9. 1946, G. Stenlid, det. S. Lundell, StenLid 1947)

\section{P. brumalis (Pers.) Fr.}

E ckerö: Skag, west of Örfjärden (1961, Anja Raita, TUR). Fi n s tröm: Apalholm (20.9.1964, Paavo Kallio, TUR). L e m 1 a n d : Flaka (7.6. 1962, Paula Siltanen, TUR); Nåtö (17.6. 1945) and Idholm (18. 6. 1945, G. Stenlid, Stenlid 1947)

\section{P. squamosus (Huds.) Fr.}

S und : Kastelholm (7-8. 1949. O.v.Sch., H)

\section{$P$. varius (Pers.) Fr.}

Geta: Bolstaholm, on Fraxinus excelsior (26. 9. 1942, R. Frey, Frey 1944). M a a $\mathrm{r}$ i a $\mathrm{n}$ h a m in a : Ytternäs (21.9. 1964, Paavo Kallio, TUR). S a $1 \mathrm{t}$ vik: Kvarnbo, on living Fraxinus 7. 1942, R. Frey, FREY 1944)

Polystictus tomentosus Fr. (Polyporus tomentosus)

Jo mala: on living Pinus silvestris (.9.1958, O.v.Sch., HFR); Möckelö (21. 8. 1958, O.v.Sch., HFR). L e m la n d: Lemböte (23.9. 1946, G. Stenlid, StenLid 1947). M a a rian ha min a: (10.8. 1958, O.v.Sch., HFR)

Pycnoporus cinnabarinus (Jacq.) Karst.

E ckerö: Skag, on Betula (7.10.1942, R. Frey, H); Skag, on Betula (7. 10. 1942, C. Cedercreutz, H). G e ta : Östergeta, Byträsk, on Betula (1943, C. Cedercreutz, H). Le m I a n d: Gripen, Granholm, on Fraxinus excelsior (1964, Aune Haakana, H)

Scutiger ovinus (Shaeff.) Bond. et Sing. (Polyporus ovinus)

G e t a : Östergeta, in coniferous forest (28.9. 1942, R. Frey, FrEY 1944)

Spongipellis spumeus (Sow.) Fr.

very rare in Finland. L e m la nd: Nåtö, on living Fraxinus (18. 9. 1946, G. Stenlid, det. S. Lundell, StenLid 1947)

Tyromyces albidus (Schaeff.) Donk

Ecke rö: Skag, on Picea abies (4. 10. 1965, L.L. \& M.K.). Finström: Mangelbo, on Picea abies (7. 10. 1965, L.L. \& M.K.). F öglö: Bråttö, on Pinus silvestris (3. 10.1965, L.L. \& M.K.); $1 \mathrm{~km}$ to the east of Sonnboda, on Picea abies (9.10.1966, L.L. \& V.H.). Jomala: Gottby, on stumps of
Picea abies (6. 10.1965, L.L. \& M.K.); Möckelö (6. 10. 1965, L.L. \& M.K.); $2 \mathrm{~km}$ to the north-west of Önningeby, on Picea abies (11.10.1966, L.L. \& M.K.); Nâtö (15.9. 1946) and Inre Korsö (17.9. 1946, G. Stenlid, Stenlid 1947, as Polyporus stipticus). Lu m parla nd: Skag, on Picea abies (7. 10. 1966, L.L. \& M.K.). Maarianhamina: on a stump of Picea abies (3.10. 1942, R. Frey, H)

T. aneirinus (Somm.) Bond. et Sing. (Det. V.K. 1967)

E ckerö: Skag, on Populus tremula (8. 10.1966, L.L. \& V.H.)

\section{T. caesius (Schrad.) Murr.}

Eckerö: Skag, on a stump of Picea abies and on Populus tremula (8. 10. 1966, L.L. \& V.H.). F i n strö m: Mangelbo (7. 10. 1965, L.L. \& M.K.); Torrbolstad (2. 10. 1965, L.L. \& M.K.); Mangelbo, Ulmus scabra stand, on an old wattled fence (10. 10. 1966, L.L. \& V.H.); near the border of Geta, on Picea abies (10. 10. 1966, L.L. \& V.H.). H a m m a r la nd: 1,5 $\mathrm{km}$ to the south-east of the church, on Picea abies (8. 10. 1966, L.L. \& V.H.). J o ma la: Ramsholm (4. 10. 1965, L.L. \& M.K.); Ramsholm W (6. 10.1965, L.L. \& M.K.); Ramsholm, on Picea abies (8. 10. 1966, L.L. \& V.H.); $1 \mathrm{~km}$ to the north of the church, on Picea abies (10.10.1966, L.L. \& V.H.); $2 \mathrm{~km}$ to the north-west of önningeby, on a fallen trunk of $B e$ tula (11. 10. 1966, L.L. \& V.H.). L e m la n d: Flaka, on Picea abies (11. 10.1966, L.L. \& V.H.); Lemböte (23.9. 1946) and Yttre Korsö (24.9. 1946, G. Stenlid, Stenlid 1947). Lum parland: Skag, on Picea abies (7. 10. 1966, L.L. \& V.H.). M a a r i a $\mathrm{n} \mathrm{ha} \mathrm{mi-}$ $\mathrm{n}$ a : Ytternäs, on coniferous tree (1.9. 1958, O.v.Sch., HFR); Ytternäs (5-6. 10. 1965, L.L. \& M.K.); Ytternäs, on Corylus (6. 10.1965, L.L. \& M.K.); Ytternäs, on Picea abies (10.10.1966, L.L. \& V.H.)

T. cinerascens (Bres.) Bond. et Sing. (? det. V.K. 1967)

G e t a : Soltuna, Getaberg, on Pinus silvestris (10. 10. 1966, L.L. \& V.H.)

$T$. erubescens (Fr.) Bond. et Sing.

$\mathrm{H}$ a m marla n d: Dräkneböle gård, on a stump of Pinus silvestris (5. 10. 1965, L.L. \& M.K.). M a a . $\mathrm{r}$ i a $\mathrm{n} \mathrm{h}$ a $\mathrm{m}$ in a: Ytternäs, on Picea abies (10.10. 1966, L.L. \& V.H.) V.K.)

T. floriformis (Quél.) Bond. et Sing. (Det. L.L. \& E ckerö: Skag (12.9.1963, Esteri Kankainen, TUR)

T. fragilis (Fr.) Donk

Föglö: Bråttö, on Pinus silvestris (3. 10.1965, L.L. \& M.K.). J o m a 1 a: Ramsholm (5.10.1965, L.L. \& M.K.)

T. lacteus (Fr.) Murr. (Det. V.K. 1967)

E ckerö: Skag, on deciduous tree (4. 10. 1965, L.L. \& M.K.). Lumparla nd: Långnäs, on a fallen trunk of Pinus silvestris (11. 10. 1966, L.L. \& V.H.)

T. "ptychogaster» (Ludw.) Donk

$\mathrm{M}$ a a rianh a min a: Ytternäs (10.9. 1958, O. v.Sch., HFR) 
T. semipileatus (Peck) Murr.

E ckerö: Skag, on Fraxinus excelsior (4.10. 1965, L.L. \& M.K.); Skag, on Fraxinus excelsior (8. 10. 1966, L.L. \& V.H.). J o ma la: Ramsholm, on Corylus avellana and Fraxinus excelsior (4. 10. 1965, and 5. 10. 1965, L.L. \& M.K.); Ramsholm, on Fraxinus excelsior (6.10.1965, L.L. \& M.K.); Ramsholm, on fallen trunks of Fraxinus excelsior (8.10.1966, L.L. \& V.H.). L u m p a r 1 a n d: Skag, on Fraxinus excelsior (7. 10. 1966, L.L. \& V.H.). M a a ri a $\mathrm{n} \mathrm{ha} \mathrm{-}$ $\mathrm{m}$ i $\mathrm{n}$ a: on deciduous tree (3. 10. 1958, O.v.Sch., HFR)

$T$. sericeo-mollis (Rom.) Bond. et Sing.

J o mal a: Ramsholm (5. 10. 1965, L.L. \& M.K.). $\mathrm{Lu}$ m a rla nd: Skag, on a burned trunk of $P i$ cea abies (?) (7. 10. 1966, L.L. \& V.H.)
T. tephroleucus (Fr.) Donk (Det. V.K. 1967)

E ckerö: Skag, on Populus tremula (8.10. 1966, L.L. \& V.H.)

T. trabeus (Rostk. s. Bourd. et Galz.) Parmasto (Det. V.K. 1967)

F in ström: Torrbolstad, on a fallen trunk of Pinus silvestris (2.10.1965, L.L. \& M.K.). Föglö : Sonnboda E., on Pinus silvestris (3. 10. 1965, L.L. \& M.K.); near Stentorpa (3.10.1965, L.L. \& M.K.). Geta: Soltuna, Getaberg, on Pinus silvestris (10. 10. 1966, L.L. \& V.H.). J o ma la: Möckelö, on rotten wood of Pinus silvestris (6.10.1965, L.L. \& M.K.). Lu m parland: Skag, on Picea abies (7. 10. 1966, L.L. \& V.H.)

\section{LITERATURE GITED}

Bondartsev, A. S., 1953: Trutovye griby Europejskoj Casti SSSR i Kaukaza (Polyporaceae of the European part of USSR, and the Caucasus) - 1106 pp. Moskva/Leningrad.

Christiansen, M. P., 1960: Danish Resupinate Fungi. Part II. Homobasidiomycetes. - Dansk Botanisk Arkiv 19 (2), 57-388.
Frey, R., 1944: Några anteckningar om basidsvampfloran på Aland. - Mem. Soc. F. Fl. Fenn. $19,9-16$.

Schulmann, O. v., 1960: Zur Kenntnis der Basidiomyceten Finnlands. - Karstenia 5, 5-99.

Stenlid, G., 1947: Några anteckningar om Ålands svampflora. - Mem. Soc. F. Fl. Fenn. 23, $82-90$. 